\title{
Screening for patients with Gaucher's disease using routine pathology results: PATHFINDER (ferritin, alkaline phosphatase, platelets) study
}

\author{
Tim Reynolds ${ }^{1}$, Anthony Wierzbicki², Volha Skrahina ${ }^{3}$, and Christian Beetz ${ }^{3}$ \\ ${ }^{1}$ University Hospitals of Derby and Burton NHS Foundation Trust \\ ${ }^{2}$ Guys \& St Thomas Hospitals \\ ${ }^{3}$ Centogene AG
}

March 15, 2021

\begin{abstract}
Aims: Lysosomal $\beta$-glucocerebrosidase (GBA) deficiency causes Gaucher disease (GD), a recessive disorder caused by bi-allelic mutations in GBA. The prevalence of GD is associated with ethnicity, but largely unknown and potentially underestimated in many countries. GD may manifest with organomegaly, bone involvement and neurological symptoms as well as abnormal laboratory biomarkers. This study attempted to screen for GD in patients using abnormal platelet, alkaline phosphatase (ALP) and ferritin results from laboratory databases. Methods: Electronic laboratory databases were interrogated using a 2-4 year time interval to identify from clinical biochemistry records patients with a phenotype of reduced platelets $(<150 \times 109 / \mathrm{L})$ and either elevated ALP $(>130 \mathrm{iu} / \mathrm{L})$ or ferritin $(>150$ (female) or $>250 \mu \mathrm{g} / \mathrm{L}(\mathrm{male}))$. The mean value over the screening window was used to reduce variability in results. A dried blood spot sample was collected for the determination of GBA activity in patients meeting these criteria. If low GBA activity was found then the concentration of the GD-specific biomarker glucosyl-sphingosine (lyso-GB1) was assayed, and the GBA gene sequenced. Results: Samples were obtained from 1058 patients; 232 patients had low GBA activity triggering further analysis. No new cases of GD with homozygosity for pathogenic variants were identified but 12 patients $(1 \%)$ were identified to be carriers of a pathogenic variant in GBA. Conclusions: Pathology databases hold routine information that can be used to screen for patients with inherited errors of metabolism. However, biochemical screening using mean platelets, ALP and ferritin has a low yield for unidentified cases of Gaucher Disease.
\end{abstract}

\section{Hosted file}

IJCP PATHFINDER Gaucher text.pdf available at https://authorea.com/users/331989/articles/ 513606-screening-for-patients-with-gaucher-s-disease-using-routine-pathology-resultspathfinder-ferritin-alkaline-phosphatase-platelets-study

\section{Hosted file}

IJCP Pathfinder Gauchers table 1.pdf available at https://authorea.com/users/331989/articles/ 513606-screening-for-patients-with-gaucher-s-disease-using-routine-pathology-resultspathfinder-ferritin-alkaline-phosphatase-platelets-study

\section{Hosted file}

IJCP Pathfinder Gauchers table 2.pdf available at https://authorea.com/users/331989/articles/ 513606-screening-for-patients-with-gaucher-s-disease-using-routine-pathology-resultspathfinder-ferritin-alkaline-phosphatase-platelets-study 